Revue internationale de l'économie sociale

Recma

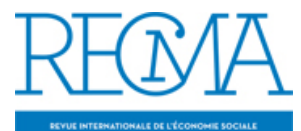

\title{
Études et ouvrages reçus
}

Numéro 305, juillet 2007

URI : https://id.erudit.org/iderudit/1021513ar

DOI : https://doi.org/10.7202/1021513ar

Aller au sommaire du numéro

Éditeur(s)

Association Recma

ISSN

0035-2020 (imprimé)

2261-2599 (numérique)

Découvrir la revue

Citer ce document

(2007). Études et ouvrages reçus. Revue internationale de l'économie sociale,

(305), 92-93. https://doi.org/10.7202/1021513ar d'utilisation que vous pouvez consulter en ligne.

https://apropos.erudit.org/fr/usagers/politique-dutilisation/ 


\section{ÉTUDES ET OUVRAGES REÇUS}

\section{L'administrateur en questions}

Coop de France, www.coopdefrance.coop, mars 2007, 2e édition, 93 p.

Un guide très complet pour aider les administrateurs de coopératives dans l'exercice de leur mission et promouvoir la gouvernance coopérative.

Les entreprises, les emplois

de l'économie sociale en Poitou-Charentes

Chiffres clés 2006

Cres Poitou-Charentes, www.cres-pch.org, mars 2007, $47 p$.

Une cartographie des 6300 établissements d'économie sociale que compte la région, soit 4600 entreprises à statut associatif, coopératif ou mutualiste.

\section{Assises régionales de l'entrepreneuriat} social, rapport de synthèse

Une réponse aux enjeux de société

Cres Provence-Alpes-Côte d'Azur, www.crespaca.org, septembre 2006, 57 p.

Une mise en perspective des interventions et débats des journées du 30 novembre et $1^{\text {er }}$ décembre 2005 pour mieux cerner le rôle de l'économie sociale face aux enjeux de société.

\section{Trajectoires associatives - Enquête} sur les facteurs de fragilité des associations Viviane Tchernonog, Jean-Pierre Vercamer, Deloitte, CNRS, université Paris-I

Panthéon-Sorbonne, mars 2006, 34 p.

Une étude centrée sur les associations employeurs soumises à l'instabilité des ressources, qui propose aussi des éléments de réponse à la fragilité.

\section{Ouvrages}

Rapport moral sur l'argent dans le monde Les entreprises de l'économie sociale en question Les grands dossiers d'éthique financière Association d'économie financière, www.aef.asso.fr, Paris, mai 2007, 449 p.

Plus de quarante auteurs contribuent à ce rapport qui interroge la pertinence des organisations de l'économie sociale face au modèle de la société anonyme et aborde des thèmes tels que la lutte contre le blanchiment ou les nouvelles fondations philanthropiques.

\section{" Nous protégeons l'infortune "}

Les origines populaires

de l'économie sociale au Québec

Martin Petitclerc, VLB éditeur, www.edvlb.com, Québec, mars 2007, 278 p. Sous la devise d'une importante société de secours mutuels, l'ouvrage montre en particulier comment la mutualité contribue, au XIX ${ }^{\mathrm{e}}$ siècle, à la formation de la classe ouvrière au Québec.

\section{L'irrésistible montée de l'économie sociale} Un projet, une culture, des valeurs Virginie Robert, collection "Monde d'aujourd'hui ", Editions Autrement, Paris, mars 2007, 95 p.

Manuel destiné à un large public pour mieux faire connaître les multiples facettes de l'économie sociale comme acteur incontournable de l'économie française. 
L'économie sociale, une alternative planétaire

Mondialiser au profit de tous

Coordonné par Thierry Jeantet

et Jean-Philippe Poulnot, Editions Charles

Léopold Mayer, Paris, février 2007, 352 p.

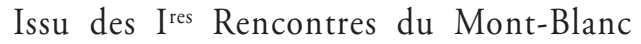
(www.rencontres-montblanc.coop), cet ouvrage réunit des contributions de différents continents et, à travers de nombreux exemples, témoigne de la diversité et de la richesse de l'économie sociale dans le monde.

\section{L'audace des entrepreneurs sociaux Concilier efficacité économique et innovation sociale \\ Virginie Seghers, Sylvain Allemand, collection "Acteurs de la société ",} Editions Autrement, Paris, janvier 2007, 239 p. Enquête approfondie qui donne la parole aux acteurs, aux institutions et aux chercheurs concernés par l'entrepreneuriat social, et que vient compléter un cahier pratique.

\section{Les banques coopératives en France} Le défi de la performance et de la solidarité Nadine Richez-Battesti, Patrick Gianfaldoni (dir.), collection "L'esprit économique ", L'Harmattan, www.librairieharmattan.com, Paris, décembre 2006, 294 p.

Une étude approfondie et complète de la finance coopérative qui montre la capacité des banques de l'économie sociale à maintenir leur efficacité tout en restant fidèles à leur mission solidaire ${ }^{(1)}$.

(1) Une note de lecture de cet ouvrage sera publiée dans le numéro 306 de la Recma.

\section{Les dynamiques de l'économie sociale et solidaire}

Jean-Noël Chopart, Guy Neyret, Daniel

Rault (dir.), collection "Recherches ", La Découverte, Paris, novembre 2006, 289 p. Issus du programme de recherche "L'économie sociale et solidaire en région ", des regards croisés et des exemples concrets qui permettent de redécouvrir l'« archipel » économie sociale.

\section{Les initiatives solidaires}

La réciprocité face au marché et à l'Etat

Laurent Gardin, Editions Erès,

www.edition-eres.com, octobre 2006, 192 p.

Fondé sur des observations en France et dans plusieurs pays européens, ce travail met en avant l'importance de la réciprocité dans le fonctionnement des initiatives solidaires.

\section{Histoire des faits économiques et sociaux de 1945 à nos jours}

Alain Beitone, Philippe Gilles,

Maurice Parodi, collection "Précis ", Dalloz, www.dalloz.fr, $3^{e}$ édition, Paris, août 2006, 503 p. La nouvelle édition de cet ouvrage de référence intègre les données macroéconomiques et les grands événements économiques, sociaux et politiques mondiaux du début du XXI ${ }^{e}$ siècle.

\section{Responsabilité sociale:}

vers une nouvelle communication des entreprises?

Patrice de la Broise, Thomas Lamarche

(édit.), Presses universitaires

du Septentrion, www.septentrion.com, 2006, $221 p$.

A partir d'une approche interdisciplinaire, l'ouvrage regroupe des contributions de chercheurs francophones, abordant par exemple les questions du bilan sociétal ou de la mesure en matière de développement durable. 